\title{
Determination of $\left|V_{u s}\right|$ from a lattice-QCD calculation of the $K \rightarrow \pi \ell \nu$ semileptonic form factor with physical quark masses
}

A. Bazavov, ${ }^{1,2}$ C. Bernard,${ }^{3}$ C.M. Bouchard,${ }^{4}$ C. DeTar, ${ }^{5}$ Daping Du, ${ }^{6}$ A.X. El-Khadra,${ }^{6,7}$ J. Foley ${ }^{5}$ E.D. Freeland, ${ }^{8}$ E. Gámiz, ${ }^{9}{ }^{*}$ Steven Gottlieb,${ }^{10}$ U.M. Heller, ${ }^{11}$ Jongjeong Kim, ${ }^{12}$ A.S. Kronfeld, ${ }^{7}$ J. Laiho, ${ }^{13,14}$ L. Levkova, ${ }^{5}$ P.B. Mackenzie, ${ }^{7}$ E.T. Neil, $,{ }^{7}, 15,16$ M.B. Oktay, ${ }^{5}$ Si-Wei Qiu, ${ }^{5}$ J.N. Simone, ${ }^{7}$ R. Sugar, ${ }^{17}$ D. Toussaint, ${ }^{12}$ R.S. Van de Water, ${ }^{7}$ and Ran Zhou ${ }^{10,7}$

(Fermilab Lattice and MILC Collaborations)

${ }^{1}$ Physics Department, Brookhaven National Laboratory, Upton, New York, USA

${ }^{2}$ Department of Physics and Astronomy, University of Iowa, Iowa, USA

${ }^{3}$ Department of Physics, Washington University, St. Louis, Missouri, USA

${ }^{4}$ Department of Physics, The Ohio State University, Columbus, Ohio, USA

${ }^{5}$ Physics Department, University of Utah, Salt Lake City, Utah, USA

${ }^{6}$ Physics Department, University of Illinois, Urbana, Illinois, USA

${ }^{7}$ Fermi National Accelerator Laboratory, Batavia, Illinois, USA

${ }^{8}$ Liberal Arts Department, School of the Art Institute of Chicago, Chicago, Illinois, USA

${ }^{9}$ CAFPE and Departamento de Física Teórica y del Cosmos, Universidad de Granada, Granada, Spain

${ }^{10}$ Department of Physics, Indiana University, Bloomington, Indiana, USA

${ }^{11}$ American Physical Society, Ridge, New York, USA

${ }^{12}$ Department of Physics, University of Arizona, Tucson, Arizona, USA

${ }^{13}$ SUPA, School of Physics and Astronomy, University of Glasgow, Glasgow, UK

${ }^{14}$ Department of Physics, Syracuse University, Syracuse, New York, USA

${ }^{15}$ Department of Physics, University of Colorado, Boulder, Colorado, USA

${ }^{16}$ RIKEN-BNL Research Center, Brookhaven National Laboratory, Upton, New York, USA

${ }^{17}$ Department of Physics, University of California, Santa Barbara, California, USA

(Dated: November 4, 2018)

We calculate the kaon semileptonic form factor $f_{+}(0)$ from lattice QCD, working, for the first time, at the physical light-quark masses. We use gauge configurations generated by the MILC collaboration with $N_{f}=2+1+1$ flavors of sea quarks, which incorporate the effects of dynamical charm quarks as well as those of up, down, and strange. We employ data at three lattice spacings to extrapolate to the continuum limit. Our result, $f_{+}(0)=0.9704(32)$, where the error is the total statistical plus systematic uncertainty added in quadrature, is the most precise determination to date. Combining our result with the latest experimental measurements of $K$ semileptonic decays, one obtains the Cabibbo-Kobayashi-Maskawa matrix element $\left|V_{u s}\right|=0.22290(74)(52)$, where the first error is from $f_{+}(0)$ and the second one is from experiment. In the first-row test of CabibboKobayashi-Maskawa unitarity, the error stemming from $\left|V_{u s}\right|$ is now comparable to that from $\left|V_{u d}\right|$.

PACS numbers: 13.20.Eb, 12.15.Hh, 12.38.Gc 
Introduction: The Cabibbo-Kobayashi-Maskawa [1] (CKM) matrix underpins all quark flavor-changing interactions in the standard model of particle physics. Symmetries reduce the number of physical parameters of this $3 \times 3$ unitary matrix to four. They can be taken to be $\left|V_{u s}\right|,\left|V_{u b}\right|,\left|V_{c b}\right|$, and $\arg \left(V_{u b}^{*}\right)$, where subscripts denote the quark flavors interacting with the $W$ boson. The focus of this Letter is to reduce the theoretical uncertainty in the first of these, in a way that sharpens the test of CKM unitarity from the first row of the matrix.

The test asks whether, or how precisely,

$$
\Delta_{u} \equiv\left|V_{u d}\right|^{2}+\left|V_{u s}\right|^{2}+\left|V_{u b}\right|^{2}-1
$$

vanishes. The CKM matrix elements are determined from, respectively, superallowed nuclear $\beta$ decays, kaon decays, and $B$-meson decays to charmless final states. A failure of the test would be evidence for phenomena beyond the standard model. As it happens, $\Delta_{u}$ and analogous tests remain in agreement with the CKM paradigm. Still, the absence of deviations provides stringent constraints on nonstandard phenomena and their energy scale [2].

Until now, the error

$$
\begin{aligned}
\left(\delta \Delta_{u}\right)^{2} & =4\left|V_{u d}\right|^{2}\left(\delta\left|V_{u d}\right|\right)^{2}+4\left|V_{u s}\right|^{2}\left(\delta\left|V_{u s}\right|\right)^{2} \\
& +4\left|V_{u b}\right|^{2}\left(\delta\left|V_{u b}\right|\right)^{2}
\end{aligned}
$$

has been dominated by the second term, because $\left|V_{u d}\right|=$ $0.97425 \pm 0.00022$ is so precise [3] (the third term is negligible). One can determine $\left|V_{u s}\right|$ via the axial-vector current, i.e., leptonic kaon decays [4-10], or via the vector current, i.e., semileptonic decays [11-15]. The current precision is at the level of $0.23-0.4 \%[9,10]$ for the former, but only $\sim 0.5 \%[14,15]$, for the latter. According to the standard model, both approaches should yield the same result, because the $W$-boson current has the structure $V-A$.

For semileptonic decays, the relation between the experimentally measured $K \rightarrow \pi \ell \nu(\gamma)$ inclusive decay width and the CKM matrix element $\left|V_{u s}\right|$, up to well known overall factors, is [16]

$$
\Gamma_{K_{l 3(\gamma)}} \propto\left|V_{u s}\right|^{2}\left|f_{+}^{K^{0} \pi^{-}}(0)\right|^{2}\left(1+\delta_{\mathrm{EM}}^{K l}+\delta_{\mathrm{SU}(2)}^{K \pi}\right) .
$$

The quantities $\delta_{\mathrm{EM}}^{K l}$ and $\delta_{\mathrm{SU}(2)}^{K \pi}$ denote long-distance electromagnetic and strong isospin-breaking corrections, respectively [16]. The latter is defined as a correction relative to the $K^{0}$ mode. The quantity needed from lattice $\mathrm{QCD}$ is the vector form factor $f_{+}(0)$, defined by

$$
\begin{aligned}
\left\langle\pi\left(p_{\pi}\right)\left|V^{\mu}\right| K\left(p_{K}\right)\right\rangle= & f_{+}^{K \pi}\left(q^{2}\right)\left[p_{K}^{\mu}+p_{\pi}^{\mu}-\frac{m_{K}^{2}-m_{\pi}^{2}}{q^{2}} q^{\mu}\right] \\
& +f_{0}^{K \pi}\left(q^{2}\right) \frac{m_{K}^{2}-m_{\pi}^{2}}{q^{2}} q^{\mu}
\end{aligned}
$$

where $V^{\mu}=\bar{s} \gamma^{\mu} u$ and $q=p_{K}-p_{\pi}$ is the momentum transfer.
We previously [14] presented a lattice-QCD calculation of $f_{+}(0)$ using the $N_{f}=2+1$ gauge-field configurations generated by the MILC Collaboration. The RBC/UKQCD Collaboration presented an independent calculation [15], using a different set of $N_{f}=2+1$ gaugefield configurations. Even though both works reduce the error on $\left|V_{u s}\right|$ from $f_{+}(0)$ to $\sim 0.5 \%$, it is still roughly two times larger than the experimental uncertainty from $\Gamma_{K_{l 3(\gamma)}}$.

Before, our dominant systematic uncertainty came from the chiral extrapolation of light-quark masses from their simulation values to the physical point [14]. Here, we reduce this uncertainty by a factor of five with data directly at the physical light-quark mass. Thus, the extrapolation becomes an interpolation. We work with a subset of the $N_{f}=2+1+1$ ensembles generated (again) by the MILC Collaboration [17]. The new ensembles use an action for the sea quarks with three-times smaller discretization effects. We now use three different lattice spacings, instead of only two. In these ensembles, the strange sea-quark masses are much better tuned than before, reducing another important uncertainty in Ref. [14]. Finally, the new ensembles include the effects of charm quarks in the sea.

Simulation details and statistical errors: We largely follow the strategy of Ref. [14]. Hence, this Letter only summarizes the main features and points out the differences. We refer the reader to Ref. [14] for details of our methodology and to Ref. [18] for technical details of the current numerical work.

We obtain the form factor using the relation [19]

$$
f_{+}(0)=f_{0}(0)=\frac{m_{s}-m_{l}}{m_{K}^{2}-m_{\pi}^{2}}\left\langle\pi\left(p_{\pi}\right)|s \bar{u}| K\left(p_{K}\right)\right\rangle .
$$

The last expression requires no renormalization and allows us to extract the form factor from three-point correlation functions with less noise than Eq. (4). The momentum of the pion, $p_{\pi}$, is adjusted via partially twisted boundary conditions $[20,21]$, such that $q^{2}=0$.

Table I shows the simulation parameters of the ensembles used here [17]. These ensembles use a one-loop Symanzik-improved gauge action for the gluons [22, 23], and the highly-improved staggered-quark (HISQ) action [24] for the $u, d, s$, and $c$ quarks in the sea. The HISQ sea quarks were simulated with the fourth-root procedure for eliminating extra quark species (often called tastes) arising from fermion doubling [25-34].

We study data at four different values of the lattice spacing. The number of configurations analyzed at $a \approx$ $0.06 \mathrm{fm}$ is too small to remove autocorrelation effects in a controlled way, so this data set is not used in the central fit but as a cross-check of discretization effects.

The strange and charmed masses are always near their physical values. In most cases, however, a better tuning of $m_{s}$ became available before computing the matrix element in Eq. (5). We have chosen the better tuned value 
TABLE I. Parameters of the $N_{f}=2+1+1$ gauge-field ensembles and correlation functions generated in this work. $N_{\text {conf }}$ is the number of configurations included, $N_{\text {src }}$ the number of time sources used on each configuration, and $L$ the spatial size of the lattice. Pion masses (fourth and fifth columns) are given in $\mathrm{MeV}$. Further information, including the light and charm quark masses, can be found in Ref. [17].

\begin{tabular}{cclcccccc}
\hline \hline$\approx a(\mathrm{fm})$ & $a m_{s}^{\text {sea }}$ & $a m_{s}^{\text {val }}$ & $m_{\pi}^{P}$ & $m_{\pi}^{\mathrm{RMS}}$ & $m_{\pi} L$ & $N_{\text {conf }}$ & $N_{\text {src }}$ \\
\hline 0.15 & 0.0647 & 0.06905 & 133 & 311 & 3.30 & 1000 & 4 \\
\hline 0.12 & 0.0509 & 0.0535 & 309 & 370 & 4.54 & 1053 & 8 \\
& 0.0507 & 0.053 & 215 & 294 & 4.29 & 993 & 4 \\
& 0.0507 & 0.053 & 215 & 294 & 5.36 & 391 & 4 \\
& 0.0507 & 0.0531 & 133 & 241 & 3.88 & 945 & 8 \\
\hline 0.09 & 0.037 & 0.038 & 312 & 332 & 4.50 & 775 & 4 \\
& 0.0363 & 0.038 & 215 & 244 & 4.71 & 853 & 4 \\
& 0.0363 & 0.0363 & 128 & 173 & 3.66 & 621 & 4 \\
\hline 0.06 & 0.024 & 0.024 & 319 & 323 & 4.51 & 362 & 4 \\
\hline \hline
\end{tabular}

for the valence quarks, hence the different values of $m_{s}^{\mathrm{val}}$ and $m_{s}^{\text {sea }}$. The up and down sea-quark masses are taken to be the same: $m_{l}=0.2 m_{s}, 0.1 m_{s}$, or $m_{s} / 27$. The last corresponds very nearly to the physical pion mass, $135 \mathrm{MeV}$. We include data at heavier-than-physical pion masses to further control the chiral-continuum fit.

While the column labeled by $m_{\pi}^{P}$ in Table I corresponds to the valence pion, the root-mean-squared pion mass, $m_{\pi}^{\text {RMS }}$, provides a measure of the dominant discretization effects, due to lattice-artifact interactions between staggered quarks of different tastes. These taste splittings, are of order $\alpha_{s}^{2} a^{2}$ for the HISQ action, where $\alpha_{s}$ is the strong coupling at a scale around $\pi / a$. They decrease rapidly with the lattice spacing, as can be seen from the difference of the fifth and fourth columns in Table I.

We obtain both hadronic matrix elements and meson energies from combined fits of two-point and three-point correlation functions. The structure of these three-point functions is the same as in Ref. [14], but here we only include moving $\pi$ data [18]. The correlation function fits include ground states, and excited and opposite-parity states [14].

Our correlation-function fits are stable under variations of the number of states, time ranges, source-sink separations, and other aspects of the fits. The central values and statistical errors are shown as a function of the light quark mass in Fig. 1, which is discussed in more detail below. Numerical values are given in Ref. [18]. Within the statistical errors of relative size $\sim 0.2-0.4 \%$, the data show no discretization effects except possibly at $0.15 \mathrm{fm}$.

Chiral interpolation and continuum extrapolation: Even though we have data at the physical quark masses, we include data at larger $m_{\pi}$. The use of chiral perturbation theory $(\chi \mathrm{PT})$ and data at different masses allows us to correct for small mistunings of the light- and strange-quark masses, as well as for partially quenched effects due to $m_{s}^{\mathrm{val}} \neq m_{s}^{\text {sea }}$. In addition, these data are very precise and help to reduce the final statistical error. Furthermore, the dominant discretization effects, are well-described by the $\chi \mathrm{PT}$ formula $[35,36]$, so they are removed when taking the continuum limit.

In $\chi \mathrm{PT}$, the form factor $f_{+}(0)$ can be written as $f_{+}(0)=1+f_{2}+f_{4}+\ldots$ In continuum $\mathrm{QCD}$, the Ademollo-Gatto theorem [37] ensures that the $\mathrm{O}\left(p^{2 i}\right)$ chiral corrections $f_{2 i}$ tend to zero in the $\mathrm{SU}(3)$ limit as $\left(m_{K}^{2}-m_{\pi}^{2}\right)^{2}$. In particular, $f_{2}$ is completely fixed in terms of well-known quantities. At finite lattice spacing, however, violations of the Ademollo-Gatto theorem arise from discretization effects in the dispersion relation needed to derive the relation in Eq. (5).

We perform the interpolation to the physical masses and the continuum using next-to-next-to-leading-order (NNLO) continuum $\chi \mathrm{PT}[38]$, supplemented by nextto-leading-order (NLO) partially quenched, staggered $\chi \mathrm{PT}$ [39]. Because we observe almost no lattice-spacing dependence in our data, discretization effects in higherloop $\chi \mathrm{PT}$ should be negligible. After removing the dominant discretization effects with $\mathrm{S} \chi \mathrm{PT}$, the remaining ones, which stem from violations of the continuum dispersion relation and higher orders taste-splitting effects, are of order $\alpha_{s} a^{2}, a^{4},\left(m_{K}^{2}-m_{\pi}^{2}\right)^{2} \alpha_{s} a^{2}$, and $\left(m_{K}^{2}-m_{\pi}^{2}\right)^{2} \alpha_{s}^{2} a^{2}$. We introduce fit parameters for these terms $-K_{1}, K_{3}$,

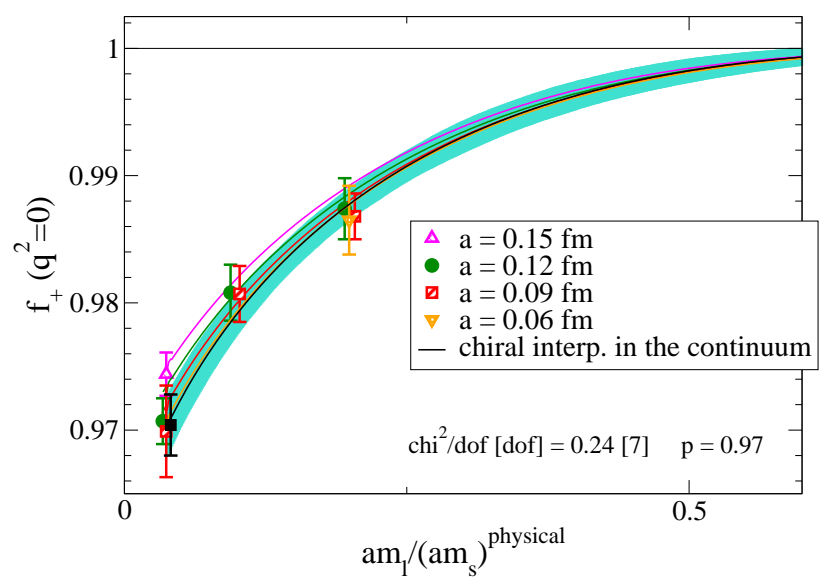

FIG. 1. Form factor $f_{+}(0)$ vs. light-quark mass. Errors shown are statistical only, obtained from 500 bootstraps. Different symbols and colors denote different lattice spacings, and the corresponding colored lines show the chiral interpolation at fixed lattice spacing. The solid black line is the interpolation in the light-quark mass, keeping $m_{s}$ equal to its physical value, and turning off all discretization effects. The turquoise error band includes statistical, discretization, and higher order chiral errors, as explained in the text. 
$K_{2}$, and $K_{2}^{\prime}$, respectively - and take the functional form

$$
\begin{gathered}
f_{+}(0)=1+f_{2}^{\mathrm{PQS} \chi \mathrm{PT}}(a)+K_{1} a^{2} \sqrt{\bar{\Delta}}+K_{3} a^{4}+f_{4}^{\mathrm{cont}} \\
+\left(m_{\pi}^{2}-m_{K}^{2}\right)^{2}\left[C_{6}+K_{2} a^{2} \sqrt{\bar{\Delta}}+K_{2}^{\prime} a^{2} \bar{\Delta}\right. \\
\left.+C_{8} m_{\pi}^{2}+C_{10} m_{\pi}^{4}\right]
\end{gathered}
$$

where $f_{2}^{\mathrm{PQS} \chi \mathrm{PT}}(a)$ is the NLO partially quenched staggered $\chi \mathrm{PT}$ expression including leading isospin corrections [40], $f_{4}^{\text {cont }}$ is the sum of the NNLO continuum chiral logarithms, and $a^{2} \bar{\Delta}$ is the average taste splitting, with $\bar{\Delta}$ used as a proxy for $\alpha_{s}^{2}$. The analytic term $C_{6}$ is related to a combination of low-energy constants in continuum $\chi \mathrm{PT}$, and $C_{8}$ and $C_{10}$ are fit parameters that parametrize chiral corrections at $\mathrm{N}^{3} \mathrm{LO}$ and $\mathrm{N}^{4} \mathrm{LO}$, respectively. We take the taste splittings from Ref. [17] and set the rest of the inputs in the same way as in Ref. [14]. The fit parameters are constrained with Bayesian techniques. We fix their prior widths using power counting arguments, except for $K_{2}^{\prime}$, where we triple the power-counting width, since it is the numerically dominant term in our fits [18]. Using Eq. (6) expressed in terms of meson masses, we interpolate to physical pion and kaon masses with electromagnetic effects removed [41, 42]: $m_{\pi^{+}}^{\mathrm{QCD}}=135.0 \mathrm{MeV}$, $m_{K^{0}}^{\mathrm{QCD}} \approx m_{K^{0}}^{\text {phys }}=497.7 \mathrm{MeV}$, and $m_{K^{+}}^{\mathrm{QCD}}=491.6 \mathrm{MeV}$. The last value enters only in $f_{2}$.

We estimate the statistical errors by generating a set of 500 pseudoensembles via the bootstrap method, and repeating the fit on each pseudoensemble. The result from the chiral and continuum interpolation/extrapolation is $f_{+}(0)=0.9704(24)$, which is shown in Fig. 1 . The fits cannot precisely determine the coefficients $K_{i}$ in Eq. (6), since only the $a \approx 0.15 \mathrm{fm}$ point appears to show any discretization effects. We examine this issue via fits with fewer parameters, including one-by-one the analytical $a^{2}$ terms in Eq. (6), and excluding higher order chiral terms (third line in Eq. (6)) to make the comparison cleaner. The results of these fits are shown in Table II. We find no difference except when all of the discretization effects are omitted. Something similar happens with the addition of higher order chiral terms to the fit function. Adding a $\mathrm{N}^{3} \mathrm{LO}$ term slightly changes the central value and increases the error from $0.9703(23)$ to $0.9704(24)$. Adding a $\mathrm{N}^{4} \mathrm{LO}$ term does not change either the central value or the error. The alternate fits with additional discretization terms and/or chiral terms show that fit errors are saturated. We thus consider the error from the chiral and continuum interpolation/extrapolation with the fit function in Eq. (6), $f_{+}(0)=0.9704(24)$, as the total statistical+discretization+chiral interpolation error. The increase in the error when adding a $\mathrm{N}^{3} \mathrm{LO}$ term, 0.0004 , gives a measure of the chiral interpolation error, five times smaller than in our previous work [14], thanks to the inclusion of data at physical quark masses. We discuss further tests of the robustness of this Bayesian error estimate strategy in Ref. [18].
TABLE II. Stability of the continuum extrapolation with omission of discretization terms, in the notation of Eq. (6).

\begin{tabular}{lccc}
\hline \hline \multicolumn{1}{c}{ Parameters omitted } & $f_{+}(0)$ & $\chi^{2} /$ dof & $p$ \\
\hline$C_{8}, C_{10}, K_{1}, K_{2}, K_{3}, K_{2}^{\prime}$ & $0.9714(12)$ & 0.27 & 0.97 \\
$C_{8}, C_{10}, K_{1}, K_{2}, K_{3}$ & $0.9703(23)$ & 0.24 & 0.97 \\
$C_{8}, C_{10}, K_{2}, K_{3}$ & $0.9703(23)$ & 0.24 & 0.97 \\
$C_{8}, C_{10}, K_{3}$ & $0.9703(23)$ & 0.24 & 0.97 \\
$C_{8}, C_{10}$ & $0.9703(23)$ & 0.24 & 0.97 \\
\hline Central fit: full Eq. (6) & $0.9704(24)$ & 0.24 & 0.97 \\
\hline \hline
\end{tabular}

Although we omit it from the chiral and continuum interpolation/extrapolation, we also show data on an ensemble with a smaller lattice spacing, $a \approx 0.06 \mathrm{fm}$, and $m_{l}^{\text {sea }}=0.2 m_{s}^{\text {sea }}$, the (orange) down-pointing triangle in Fig. 1. It lies on top of the results for the other lattice spacings, confirming that discretization effects are much smaller than statistical errors. The same conclusion follows from the fact that the red line in Fig. 1, for $a \approx 0.09 \mathrm{fm}$, is very close to the continuum one. The remaining significant sources of systematic uncertainty are given in Table III. We estimate the error due to including partially quenched effects only at one loop by the shift in the final result when using $m_{s}^{\text {val }}$ or $m_{s}^{\text {sea }}$ in the NNLO chiral logarithmic function, $f_{4}^{\text {cont }}$. To convert dimensionful quantities from lattice to physical units, we use the scale $r_{1}=0.3117(22) \mathrm{fm}$ [43] obtained from the static-quark potential $[44,45]$. The form factor, being a dimensionless quantity, depends on the scale only via the input parameters. Propagating the uncertainty in the scale through to $f_{+}(0)$ yields the entry shown in Table III. For an estimate of the finite volume error we compare our data obtained with two different spatial volumes and other parameters at $a \approx 0.12 \mathrm{fm}$ fixed. The difference is about half of the statistical error, so we take the finite volume error to be the full size of the statistical error. Finally, we estimate the error from the NNLO and higher order isospin corrections to the $K^{0} \pi^{+}$mode by taking twice the difference between the NNLO contribution to $f_{+}(0)$ with and without isospin corrections [46]. See Ref. [18] for more details.

Final result and conclusions: Our final result for the vector form factor is

$$
f_{+}(0)=0.9704(24)(22)=0.9704(32),
$$

where the first error is from the chiral-continuum fit, and the second the sum in quadrature of the other systematic errors listed in Table III. This result is the most precise calculation of $f_{+}(0)$ to date and the first to include data at physical light-quark masses. It agrees with the previous results of Refs. [14, 15], with a reduced total uncertainty of $0.33 \%$.

Using the latest average of experimental results for $K$ semileptonic decays, $\left|V_{u s}\right| f_{+}(0)=0.2163(5)$ [47], and the form factor in Eq. (7), one obtains

$$
\left|V_{u s}\right|=0.22290(74)_{f_{+}(0)}(52)_{\text {expt }}=0.22290(90) .
$$


TABLE III. Error budget for $f_{+}(0)$ in percent.

\begin{tabular}{lc}
\hline \hline Source of uncertainty & Error $f_{+}(0)(\%)$ \\
\hline Stat. + disc. + chiral inter. & 0.24 \\
$m_{s}^{\text {val }} \neq m_{s}^{\text {sea }}$ & 0.03 \\
Scale $r_{1}$ & 0.08 \\
Finite volume & 0.2 \\
Isospin & 0.016 \\
\hline Total Error & 0.33 \\
\hline \hline
\end{tabular}

The unitarity test becomes

$$
\Delta_{u}=-0.00115(40)_{V_{u s}}(43)_{V_{u d}},
$$

i.e., the error on $\Delta_{u}$ from $\left|V_{u s}\right|$ is now slightly smaller than that from $\left|V_{u d}\right|$. Combining the two errors, one sees $\mathrm{a} \sim 2 \sigma$ tension with unitarity. Recall that the semileptonic decay proceeds through the vector current; the uncertainty of $\left|V_{u s}\right| /\left|V_{u d}\right|$ from the axial-vector current, via leptonic pion and kaon decays and the ratio $f_{K} / f_{\pi}$ [10] already results in a value of $\Delta_{u}$ with smaller error. As emphasized above, it is important to carry out the test with both currents.

In summary, with the HISQ $N_{f}=2+1+1$ ensembles, we have reduced the uncertainties on $\left|V_{u s}\right|$ from the chiral interpolation and discretization effects. The main remaining sources of error are Monte Carlo statistics and finite-volume effects. In order to reach the final target of $0.2 \%$ precision required by experiment, we are increasing statistics and deriving the finite-volume corrections at one-loop in partially quenched staggered $\chi \mathrm{PT}$ with twisted boundary conditions [48].

Acknowledgements: We thank Christine Davies for useful discussions. We thank Johan Bijnens for making his NLO partially quenched $\chi \mathrm{PT}$ and NNLO full QCD $\chi \mathrm{PT}$ codes available to us. A.X.K. thanks the Fermilab theory group for hospitality while this work was finalized. Computations for this work were carried out with resources provided by the USQCD Collaboration, the Argonne Leadership Computing Facility and the National Energy Research Scientific Computing Center, which are funded by the Office of Science of the United States Department of Energy; and with resources provided by the National Center for Atmospheric Research, the National Center for Supercomputing Applications, the National Institute for Computational Science, and the Texas Advanced Computing Center, which are funded through the National Science Foundation's Teragrid/XSEDE and Blue Waters Programs. This work was supported in part by the U.S. Department of Energy under Grants No. DE-FG02-91ER40628 (C.B.), No. DE-FC02-06ER41446 (C.D., J.F., L.L., M.B.O.), No. DE-FG02-91ER40661 (S.G., R.Z.), No. DOE DEFG02-13ER42001 (D.D., A.X.K.), No. DE-FG02-04ER41298 (J.K., D.T.); by the National Science Foundation under Grants No. PHY-1067881, No. PHY-0757333,
No. PHY-0703296 (C.D., J.F., L.L., M.B.O.), No. PHY0757035 (R.S.); by the URA Visiting Scholars' program (A.X.K.); by the Science and Technology Facilities Council and the Scottish Universities Physics Alliance (J.L.); by the MINECO (Spain) under Grants FPA2010-16696, FPA2006-05294, and Ramón y Cajal program (E.G.); by Junta de Andalucía (Spain) under Grants FQM101 and FQM-6552 (E.G.); and by European Commission under Grant No. PCIG10-GA-2011-303781 (E.G.). This manuscript has been co-authored by an employee of Brookhaven Science Associates, LLC, under Contract No. DE-AC02-98CH10886 with the U.S. Department of Energy. Fermilab is operated by Fermi Research Alliance, LLC, under Contract No. DE-AC02-07CH11359 with the U.S. Department of Energy.

* megamiz@ugr.es

[1] N. Cabibbo, Phys. Rev. Lett. 10, 531 (1963); M. Kobayashi and T. Maskawa, Prog. Theor. Phys. 49, 652 (1973).

[2] V. Cirigliano, J. Jenkins and M. González-Alonso, Nucl. Phys. B 830 (2010) 95 [arXiv:0908.1754 [hep-ph]].

[3] J. C. Hardy and I. S. Towner, Phys. Rev. C 79, 055502 (2009) [arXiv:0812.1202 [nucl-ex]].

[4] E. Follana et al. [HPQCD and UKQCD Collaborations], Phys. Rev. Lett. 100, 062002 (2008) [arXiv:0706.1726 [hep-lat]].

[5] S. Dürr et al., Phys. Rev. D 81, 054507 (2010) [arXiv:1001.4692 [hep-lat]].

[6] Y. Aoki et al. [RBC and UKQCD Collaborations], Phys. Rev. D 83, 074508 (2011) [arXiv:1011.0892 [hep-lat]].

[7] A. Bazavov et al. [MILC Collaboration], PoS LATTICE2010, 074 (2010) [arXiv:1012.0868 [hep-lat]].

[8] J. Laiho and R. S. Van de Water, PoS LATTICE2011, 293 (2011) [arXiv:1112.4861 [hep-lat]].

[9] A. Bazavov et al. [MILC Collaboration], Phys. Rev. Lett. 110, 172003 (2013) [arXiv:1301.5855 [hep-ph]].

[10] R. J. Dowdall, C. T. H. Davies, G. P. Lepage and C. McNeile [HPQCD Collaboration], Phys. Rev. D 88, 074504 (2013) [arXiv:1303.1670 [hep-lat]].

[11] V. Lubicz et al. [ETM Collaboration], Phys. Rev. D 80 (2009) 111502 [arXiv:0906.4728 [hep-lat]].

[12] P. A. Boyle et al. [RBC/UKQCD Collaboration], Eur. Phys. J. C 69 (2010) 159 [arXiv:1004.0886 [hep-lat]].

[13] T. Kaneko et al. [JLQCD Collaboration], PoS LATTICE2012, 111 (2012) [arXiv:1211.6180 [hep-lat]].

[14] A. Bazavov et al. [Fermilab Lattice and MILC Collaborations], Phys. Rev. D 87, 073012 (2013) [arXiv:1212.4993 [hep-lat]].

[15] P. A. Boyle, J. M. Flynn, N. Garron, A. Jüttner, C. T. Sachrajda, K. Sivalingam, and J. M. Zanotti [RBC/UKQCD Collaboration], JHEP 1308, 132 (2013) [arXiv:1305.7217 [hep-lat]].

[16] V. Cirigliano, G. Ecker, H. Neufeld, A. Pich and J. Portolés, Rev. Mod. Phys. 84, 399 (2012) [arXiv:1107.6001 [hep-ph]].

[17] A. Bazavov et al. [MILC Collaboration], Phys. Rev. D 82, 074501 (2010) [arXiv:1004.0342 [hep-lat]]; A. Baza- 
vov et al. [MILC Collaboration], Phys. Rev. D 87, 054505 (2013) [arXiv:1212.4768 [hep-lat]].

[18] E. Gámiz et al. [Fermilab Lattice and MILC Collaborations], PoS LATTICE2013, $395 \quad$ (2013) [arXiv:1311.7264 [hep-lat]].

[19] H. Na, C. T. H. Davies, E. Follana, G. P. Lepage, J. Shigemitsu [HPQCD Collaboration], Phys. Rev. D 82 (2010) 114506 [arXiv:1008.4562 [hep-lat]].

[20] P. F. Bedaque, J.-W. Chen, Phys. Lett. B 616, 208-214 (2005) [hep-lat/0412023].

[21] C. T. Sachrajda and G. Villadoro, Phys. Lett. B 609, 73 (2005) [hep-lat/0411033].

[22] M. Lüscher and P. Weisz, Phys. Lett. B 158, 250 (1985).

[23] A. Hart et al. [HPQCD Collaboration], Phys. Rev. D 79, 074008 (2009) [arXiv:0812.0503 [hep-lat]].

[24] E. Follana et al. [HPQCD and UKQCD Collaborations], Phys. Rev. D 75, 054502 (2007) [hep-lat/0610092].

[25] E. Marinari, G. Parisi and C. Rebbi, Nucl. Phys. B 190, 734 (1981).

[26] S. Dürr and C. Hoelbling, Phys. Rev. D 69, 034503 (2004) [hep-lat/0311002].

[27] E. Follana, A. Hart and C.T.H. Davies [HPQCD and UKQCD Collaborations], Phys. Rev. Lett. 93 (2004) 241601 [hep-lat/0406010].

[28] S. Dürr, C. Hoelbling and U. Wenger, Phys. Rev. D 70, 094502 (2004) [hep-lat/0406027].

[29] Y. Shamir, Phys. Rev. D 71, 034509 (2005) [heplat/0412014]; Phys. Rev. D 75, 054503 (2007) [heplat/0607007].

[30] C. Bernard, Phys. Rev. D 73, 114503 (2006) [heplat/0603011].

[31] C. Bernard, M. Golterman, Y. Shamir and S. R. Sharpe, Phys. Lett. B 649, 235 (2007) [hep-lat/0603027].

[32] C. Bernard, M. Golterman and Y. Shamir, Phys. Rev. D 77, 074505 (2008) [arXiv:0712.2560 [hep-lat]].
[33] D. H. Adams, Phys. Rev. D 77, 105024 (2008) [arXiv:0802.3029 [hep-lat]].

[34] G. C. Donald, C. T. H. Davies, E. Follana and A. S. Kronfeld, Phys. Rev. D 84, 054504 (2011) [arXiv:1106.2412 [hep-lat]].

[35] W.-J. Lee and S. R. Sharpe, Phys. Rev. D 60, 114503 (1999) [hep-lat/9905023].

[36] C. Aubin and C. Bernard, Phys. Rev. D 68, 034014 (2003) [arXiv:hep-lat/0304014]; Phys. Rev. D 68, 074011 (2003) [arXiv:hep-lat/0306026].

[37] M. Ademollo and R. Gatto, Phys. Rev. Lett. 13, 264 (1964).

[38] J. Bijnens and P. Talavera, Nucl. Phys. B 669, 341 (2003) [hep-ph/0303103].

[39] C. Bernard, J. Bijnens and E. Gámiz, arXiv:1311.7511 [hep-lat].

[40] J. Gasser and H. Leutwyler, Nucl. Phys. B 250, 517 (1985).

[41] S. Aoki et al. [FLAG Working Group], arXiv:1310.8555 [hep-lat].

[42] S. Basak et al. [MILC Collaboration], PoS CD12, 030 (2013) [arXiv:1301.7137 [hep-lat]].

[43] A. Bazavov et al. [Fermilab Lattice and MILC Collaborations], Phys. Rev. D 85, 114506 (2012) [arXiv:1112.3051 [hep-lat]].

[44] C. W. Bernard et al., Phys. Rev. D 62, 034503 (2000) [hep-lat/0002028].

[45] R. Sommer, Nucl. Phys. B 411, 839 (1994) [heplat/9310022].

[46] J. Bijnens and K. Ghorbani, arXiv:0711.0148 [hep-ph].

[47] M. Moulson, "Testing the Standard Model with Kaon Decays," in CIPANP 2012, edited by B. Fleming (AIP, Melville, NY, 2013) [arXiv:1209.3426 [hep-ex]].

[48] C. Bernard, J. Bijnens and E. Gámiz, in preparation. 